\title{
QUALIDADE DE VIDA DOS PROFISSIONAIS DE SAÚDE EM AMBIENTE HOSPITALAR
}

\author{
Viviane Santos Santana ${ }^{a}$ \\ Alexandre Gomes Feitosa ${ }^{b}$ \\ Lorena Barreto Arruda Guedes ${ }^{c}$ \\ Noely Bastos Brito Sales ${ }^{d}$
}

\begin{abstract}
Resumo
Introdução: A Qualidade de vida (QV) é um tema complexo e bastante discutido na literatura sendo definida pela Organização Mundial de Saúde (OMS), como a "percepção do indivíduo de sua posição na vida no contexto da cultura e sistemas de valores nos quais ele vive e em relação aos seus objetivos, expectativas, padrões e preocupações". O ambiente hospitalar é apontado para os profissionais de saúde como insalubre e estressante, expondo-os a diversos fatores físicos e mentais. Este trabaIho tem como objetivo sistematizar o conhecimento a respeito da QV dos profissionais de saúde em ambiente hospitalar. Metodologia: Trata-se de uma revisão de literatura realizada com acesso à base de dados Medline e à biblioteca virtual Pubmed. As publicações referentes ao período compreendido entre 2009 a outubro de 2013, incluídos apenas os artigos que abordassem a QV dos profissionais de saúde no ambiente hospitalar. Foram excluídos artigos que relatassem à QV fora do contexto hospitalar, ou que abordassem a QV de acadêmicos e aqueles associados a patologias. Quatro artigos julgados relevantes localizados antes do período definido como critério de inclusão. Resultados: Foram selecionados 16 artigos, sendo treze na língua portuguesa, um na língua inglesa e dois em espanhol que preencheram os critérios inicialmente propostos e lidos na íntegra. Conclusão: Os estudos demonstram que os fatores físicos e mentais possuem impactos diretos na QV dos profissionais de saúde no âmbito hospitalar, causando diversos danos a sua saúde e apresentando-se de forma negativa no desempenho de suas atividades diárias.
\end{abstract}

Palavras-chave: Qualidade de vida; Qualidade de vida no trabalho; Profissionais de saúde; Hospital; Saúde do trabalhador; Questionários.

\footnotetext{
Autor correspondente:

a. Fisioterapeuta, pós-graduanda em Fisioterapia Hospitalar pela Escola Bahiana de Medicina e Saúde Pública (EBMSP).

b. Fisioterapeuta, pós-graduando em Fisioterapia Hospitalar pela Escola Bahiana de Medicina e Saúde Pública (EBMSP).

c. Fisioterapeuta, docente da pós- graduação em Fisioterapia Hospitalar pela Escola Bahiana de Medicina e Saúde Pública (EBMSP).

d. Fisioterapeuta, pós graduanda em Gestão Clínica das Regiões do SUS pelo Hospital Sírio Libanês e Ministério da Saúde)
} 


\title{
QUALITY OF LIFE OF HEALTH IN HOSPITAL ENVIRONMENT
}

\begin{abstract}
Introduction: Quality of life ( $Q O L$ ) is a complex and much discussed topic in the literature is defined by the World Health Organization ( WHO ), as the "individual's perception of their position in life in the context of culture and value systems in which they live and in relation to their goals, expectations, standards and concerns ". The hospital environment is targeted to health professionals as unhealthy and stressful, exposing them to various physical and mental factors. This paper aims to systematize knowledge about the QOL of health professionals in the hospital setting. Methodology: This is a literature review with access to the database Medline and Pubmed virtual library. Publications relating to the period from 2009 to October 2013, included only those articles that addressed QOL of health professionals in the hospital setting. Articles who reported the QOL outside the hospital context, or that addressed QOL of academics and those associated with pathologies were excluded. Four articles deemed relevant located before the period defined as inclusion criteria. Results: 16 articles were selected, thirteen in Portuguese, one in English and two in Spanish that met the criteria originally proposed and read in their entirety. Conclusion: These studies demonstrate that the physical and mental factors have a direct impact on the QOL of health professionals in hospitals, causing extensive damage your health and presenting themselves negatively in the performance of their daily activities. Keywords: Quality of life; Quality of work life; Health care professionals; Hospital; Occupational health.
\end{abstract}

\section{INTRODUÇÃO}

A qualidade de vida (QV) é um tema complexo. (1) É definida pela Organização Mundial de Saúde (OMS), como a: "percepção do indivíduo de sua posição na vida no contexto da cultura e sistemas de valores nos quais ele vive e em relação aos seus objetivos, expectativas, padrões e preocupações". ${ }_{(1,2,6,8,12)}$ Por este motivo, é bastante discutida na literatura. ${ }^{(1-12)}$

No Brasil, a preocupação com a questão da saúde dos trabalhadores hospitalares iniciou-se na década de 70, quando pesquisadores da Universidade de São Paulo (USP) enfocaram a saúde ocupacional em trabalhadores hospitalares. Entretanto, somente na década de 90 foram levados em conta aspectos éticos e psíquicos do trabalho na área de saúde. Apesar desse fato, as doenças ou queixas não relacionadas ao trabalho continuam sujeitas a uma análise mais apurada para exclusão de seu nexo causal relacionado ao processo de trabalho. ${ }^{(13)}$

Atualmente a sobrecarga física e emocional durante a assistência aos pacientes internados no ambiente hospitalar, é muitas vezes negligenciada pelos profissionais de saúde. ${ }^{(14)}$ Vale salientar que o ambiente hospitalar é apontado como insalubre ${ }^{(10)}$ e estressante. ${ }^{(15)}$ É neste ambiente laboral que a equipe de saúde está frequente a exposição de diversos fatores, comprometedores de suas saúdes física e mental, como lidar com a dor, o sofrimento e a morte, também, pelo sistema de turnos contínuos ou de trabalho em turnos ininterruptos de revezamento e prestação de serviços durante 24 horas diárias, nos sete dias da semana, somado a 
transição entre turnos para passagem do plantão. (16) O impacto negativo na saúde física e mental, deve-se também a falta de atenção e tempo para assuntos relacionados à atuação, deste profissional, como indivíduo inserido em meio social. Desta maneira, cuidados são necessários a fim de evitar, por exemplo, a baixa produtividade e ou doenças crônicas cujas consequências podem prejudicar a QV destes profissionais. ${ }^{(4,7,10)}$

Nesse contexto, conforme a Constituição Federal e o propósito do Ministério da Saúde, a saúde do trabalhador, têm como meta a promoção da saúde dos indivíduos e das equipes de trabalho. É dever do Estado à promoção de condições indispensáveis ao seu pleno exercício, garantir através de formulação e execução de políticas econômicas e sociais, a redução de riscos de doenças no estabelecimento de trabalho, também, criar condições que assegurem acesso universal e igualitário as ações e aos serviços para sua promoção, proteção e recuperação. ${ }^{(17)}$ Os profissionais de saúde necessitam e tem direito ao bem estar e QV, para isto, é necessário que se promova saúde, dando condições, orientação e treinamento para que possam desempenhar suas atividades, sem que esta the cause qualquer dano. ${ }^{(9)}$

Com base na relevância temática vale ressaltar, que ainda são poucos os estudos em relação à QV dos profissionais de saúde e ambiente hospitalar. Assim, o objetivo deste artigo foi sistematizar o conhecimento a respeito da QV dos profissionais de saúde em ambiente hospitalar.

\section{MATERIAIS E MÉTODOS}

Trata-se de uma revisão de literatura realizada com acesso a base de dados Medline e na biblioteca virtual Pubmed. As publicações referentes ao período compreendido entre 2009 a outubro de 2013 foram selecionadas, contudo, quatro artigos de período anterior do referido foram utilizados pela relevância dos mesmos. As palavras-chave utilizadas foram qualidade de vida, qualidade de vida no trabalho, profissionais de saúde, hospital, ambiente de trabalho, saúde do trabalhador, questionários e seus correlatos em inglês e espanhol.

Os critérios de inclusão definidos para a seleção do presente trabalho foram artigos que abordassem a QV dos profissionais de saúde no ambiente hospitalar. Os estudos que relatassem a QV em acadêmicos, aqueles referentes à $\mathrm{QV}$ associados a patologias, artigos referentes ao ambiente de trabalho fora do contexto hospitalar, foram considerados inadequados para realização deste trabalho.

\section{RESULTADOS}

Nas bases de dados foram encontrados 235 artigos na base de dados Medline, 95 na biblioteca virtual Pubmed e 30 estudos em consultas manuais nas referências dos artigos. Após a leitura dos títulos, notou-se que alguns deles se repetiram e outros não preenchiam os critérios deste estudo. Foram selecionados 60 artigos para a leitura do resumo e excluídos os que não diziam respeito ao propósito do trabalho, sendo a maior quantidade de exclusões referentes à $\mathrm{QV}$ associados a patologias. Após a leitura dos resumos, foram selecionados 16 artigos, que foram lidos na íntegra, sendo 13 na língua portuguesa, um na língua inglesa e dois em espanhol que preencheram os critérios inicialmente propostos.

A Tabela 1 sintetiza os resultados encontrados nos artigos selecionados para este estudo de revisão.

\section{DISCUSSÃO}

A referida pesquisa procurou avaliar a QV de profissionais de saúde que atuam em unidade hospitalar através dos resultados de estudos relevantes sobre o tema, uma vez que este parâmetro, apesar de complexo e nos tempos atuais ter sido melhor discutido na literatura, necessita-se ampliar as discussões, devendo estar vinculado à prevenção, controle e planejamento pessoal e um programa educacional em saúde. Os achados na literatura 
demonstram alguns estudos descritivos que avaliaram a QV. Entretanto, segundo Souza \& Stancato 2011," faltam publicações e informações sobre a QV entre os profissionais de saúde, bem com, sobre a QV destes que exercem a função em instituição hospitalar.

Pautado nas considerações anteriores em consonância com informações dos 16 artigos incluídos, detectou-se que ocorre um decréscimo da QV dos profissionais que exercem função hospitalar em circunstâncias de estarem expostos a riscos eminentes da profissão ${ }^{(4,11,18,19-21)}$ sendo indicado avaliar modificações através de uma abordagem multidisciplinar na identificação e monitorização de agentes estressores, proporcionar ambiente de trabalho seguro, com riscos reduzidos e motivar a equipe, o que é extremamente importante para estes trabalhadores, pois um dos principais problemas de saúde destes está relacionado ao ambiente insalubre e estressante. ${ }^{(10,16)}$

Os resultados dos estudos reportam a equipe de enfermagem, ressaltando esta como maior representatividade de trabalhadores no ambiente hospitalar, sendo o nível técnico apontado com percentual superior quando comparado à outras profissões de saúde, ${ }^{(3,11,22-24)}$ o que remete à importância desses profissionais que atuam com a equipe multidisciplinar na promoção de saúde. Sendo estes os que apresentam maior nível de desconforto físico e emocional quando comparados aos médicos em pesquisa realizada por Ansoelaga et al. 2011, ${ }^{(18)}$ em consonância com trabalhos similares. Diferente destes estudos, Ferreira \& Oliveira em $2007^{(7)}$ avaliaram a QV de 64 fisioterapeutas, devido à existência de fatores de risco inerentes à profissão e por ser o profissional que trata da funcionalidade e QV do outro. Já Martinez et al. $2007^{(25)}$ em seu estudo mediu a QV de 109 médicos residentes do Hospital Regional de Motril, ressaltando a dimensão enquanto profissional com especialidade de emergência. Enquanto Tong et al $2012^{(19)}$ realizaram sua pesquisa com 213 médicos de pouca a vasta experiência.
Grande parte da amostra das pesquisas em relação ao perfil dos envolvidos tem predomínio de gênero feminino. $(3,4,7,10,12,18,20,22,24-27)$ Diferente desses resultados, Tong et al. $2012^{(19)} \mathrm{em}$ seu trabalho encontraram um percentual de $55 \%$ masculino, podendo estar associado ao fato da amostra conter médicos com mais tempo de experiência, sendo que antigamente formavam-se com maior prevalência indivíduos homens.

No que diz respeito ao estado civil, os estudos apontam para os profissionais casados. $(3,4,7,11,12,20,23,24,27)$. Diferentemente, Bastos et al. $2009^{(21)}$ verificaram a QV de 116 profissionais de um Hospital de Porto Alegre, destes 55,7\% eram solteiros, bem como a pesquisa apresentada por Sales et al. $2010^{(10)}$ através da análise de 27 profissionais da Unidade de Terapia Intensiva - UTI e Unidade de Internação Não Especificada do Hospital Filantrópico de Minas Gerais em que os acha dos demonstram a prevalência de $51,95 \%$ solteiros.

Com base nos dados das referências adotadas, quanto à faixa etária, a idade média varia entre 33 e 35 anos. ${ }^{(3,4,11,21,23,24,26)}$ Demonstra que os profissionais buscam trabalhar anteriormente à terceira idade, pois acreditam que ao longo dos anos, ocorre redução da vitalidade e indisposição para realização de projetos de vida.(4) Oliveira \& Chaves-Maia 2008, (20) observaram a faixa etária amostral predominantemente acima de 45 anos (47,3\%), divergindo da grande maioria dos estudos já realizados que tratam sobre esse tema.

Segundo Sales et al. 2010,(10) ao analisarem a carga horária, dos 27 profissionais estudados grande parte, $13(48,1 \%)$ exerciam função em mais de um turno, como confirmado pela maioria dos autores sobre a jornada dupla ou tripla dos trabalhadores. ${ }^{(3,12,23,24)}$ Enquanto Carvalho \& Souza $2011^{(4)}$ constataram que os médicos e a equipe de enfermagem apresentam carga horária maior alcançando a média de 49 horas/semanais. Oliveira \& Chaves-Maia $2008^{(20)}$ acrescentam ainda que os dias de folga que dispõem para se dedicar à vida pessoal são dois dias para $54,7 \%$ dos sujeitos e $26,3 \%$ contam com somente um dia. Tais dados comprovam 
a jornada dupla ou tripla de trabalho, levando a um comprometimento significante da QV.

Alguns analisaram o aspecto nível educacional dos trabalhadores que possuem ensino superior completo, constatando que um percentual acima de $60 \%$ eram pós-graduados. ${ }^{(10,12,27)}$ Kimura \& Dirley $2009^{(27)}$ complementam que $68,5 \%$ possuíam especialização, poucos tinham completado mestrado $(9,1 \%)$ ou doutorado $(1,2 \%)$. Todavia, os autores Rios, Barbosa \& Belasco 2010 ${ }^{(23)}$ desenvolveram uma pesquisa com 266 técnicos e auxiliares de enfermagem de hospital privado de São Paulo, chegou a porcentagem de $33,1 \%$ do grupo cursando nível superior e 7,9\% já haviam concluído, sendo que $97 \%$ do total destes optou pela graduação em enfermagem.

Um decréscimo na QV foi observado para os profissionais mais experientes comparados aos de menor tempo de atuação em ambiente hospitalar, com uma média variando entre 6,6 e 13 anos, Bastos et al 2009 ${ }^{(21)}$ e Motcke et al. 2003, ${ }^{(24)}$ respectivamente. Para Fogaça, Carvalho \& Nogueira-Martins 2010, ${ }^{(26)}$ a sobrecarga nos trabalhadores da UTI,

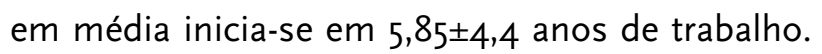
Em contraponto, Tong et al. 2012,(19) em sua análise com médicos residentes e médicos mais experientes, a variante tempo de serviço, determinou uma variação entre estes profissionais, sendo a população de residentes mais acometidas pelo desgaste físico e baixa QV. Tal divergência pode ser refletida em decorrência da fase de alto nível de estresse em período de imersão plena na atividade profissional, enfrentamento de situações complexas, que requerem soluções imediatas, de difícil controle, seja pela gravidade dos quadros clínicos, ou pelas ocorrências e limitações organizacionais.

Forte correlação é apontada entre diversos estudos ao relacionar a UTI como unidade de atuação profissional dentro do ambiente hospitalar com o déficit de QV. Como observado no trabalho de Fogaça, Carvalho \& Nogueira-Martins $2010^{(26)}$ constituído por médicos e enfermeiros da UTI pediátrica e Neonatal da Universidade Federal de São Paulo/Escola Paulista de Medicina (UNIFESP/EPM) que revelou um desgaste físico e mental, reflexo do local insalubre, da complexidade dos casos, demandas de pacientes e constante manuseio dos mesmos e consequente sobrecarga de trabalho. Embora escassas as publicações que confrontam o nível de estresse e a QV dos profissionais lotados em enfermarias com relação aqueles das UTI's, Sales et al $2010^{(10)}$ ao estudar 27 profissionais - enfermeiros, médicos e fisioterapeutas - de setores abertos, mostra que atuar nesses locais, muitas vezes, são mais estressantes quando comparados aos setores fechados, apontando como fatores desencadeantes o ritmo acelerado de trabalho e a fala de vagas em UTI, sendo os pacientes graves admitidos em enfermarias que não estão preparadas para lidar com esses casos.

$\mathrm{Na}$ mensuração da QV em diversos estudos epidemiológicos com trabalhadores de saúde em ambiente hospitalar têm sido aplicado o questionário desenvolvido pelo Grupo de Qualidade de Vida da Organização Mundial de saúde - OMS, a versão abreviada WHOQoL-bref (3,7,10-12,21-23) Este instrumento é composto de 26 questões que abrangem quatro domínios: (I) físico, (II) psicológico, (III) relações sociais e (IV) meio ambiente. Destes estudos, quatro apresentaram baixos escores para o domínio I, (10-12,23) onde Souza \& Stancato $2011^{(11)}$ atribuem esses agravos ao desgaste físico consequente à sobrecarga de trabalho, ocasionando, cansaço, fadiga, insônia e redução das atividades de vida diárias e laborais.

Para Sales et al. $2010^{(10)}$ foi constatado comprometimento no domínio II que abrange os aspectos de dar sentido e aproveitar a vida, a concentração, a aparência física, a satisfação consigo mesmo e a frequência de sentimentos negativos. Ferreira \& Oliveira $2010^{(7)}$ demonstraram baixo escore no domínio III, caracterizado pelo apoio dos amigos, vida sexual e relacionamento com pessoas próximas ou não. O comprometimento no domínio IV foi observado em quatro estudos, ${ }^{(3,11,21,23)}$ podendo estar relacionado ao ambiente de trabalho, á execução hierárquica e ao envolvimento com os pacientes, levando à fragilidades físicas e emocionais. Diferenças encontradas na pesquisa de Silva \& Camargo em 2012 ${ }^{(22)}$ através do WHOQoL-bref, 
revelou uma avaliação positiva na QV do grupo estudado $(69,7 \%)$, entretanto não apresenta os escores dos domínios, dificultando uma análise criteriosa.

Ao comparar os estudos que utilizaram o traduzido e validado instrumento na língua portuguesa, o Short Form-36 (SF-36) têm-se certa variabilidade. $(4,24)$ Trata-se de um questionário multidimensional composto de 36 itens, divididos em oito domínios: capacidade funcional, aspectos físicos, dor estado geral de saúde, vitalidade, aspectos sociais, aspectos emocionais e saúde mental. O resultado é apresentado em forma de escore final com variação de o a 100. Quanto mais próximo de o, pior o estado de saúde.

Para Carvalho \& Souza 2011,(4) a vitalidade apresentou pior escore $(69,85)$, enquanto para Motcke et al 2003 com maior comprometimento destacase a dor física com valor médio de 50. A Singapore version do SF-36 (SF-36v2) foi utilizada por Tong et al. 2012, ${ }^{19)}$ sendo constatado menores pontuações em todos os domínios, exceto para o funcionamento físico.

Existem na literatura algumas pesquisas que utilizaram instrumentos específicos de Qualidade de Vida no Trabalho (QVT). ${ }^{(18,25,27)}$ Kimura et al. 2009, ${ }^{(27)}$ através do uso do instrumento reduzido de QVT em trabalho realizado na cidade de São Paulo com participação de enfermeiros, apresentaram resultados adequados no que diz respeito à percepção dos profissionais quanto ao seu trabaIho. Contradizendo o referido estudo, Martínez et al. 2007, ${ }^{(25)}$ na Espanha, utilizando o Questionaire Professional (CVP-35), observou baixa percepção e desmotivação no enfrentamento das cargas de trabalho impostas pelos gestores.

Oliveira \& Chaves-Maia $2008^{(20)}$ citam que no ambiente hospitalar são encontradas não apenas pessoas em tratamento por patologias variadas, como também profissionais exercendo plenamente suas atividades, apesar de necessitar de cuidados pela sua precária situação de saúde física e mental. Esta inicialmente apresentando-se com pequenos distúrbios até chegar a doença propriamente dita.
Quando relacionados os sintomas psicofísicos, foram evidenciados por alguns autores com maior ênfase os distúrbios osteomioarticulares, estando relacionados principalmente às queixas de dores na coluna, ${ }^{(7,1123)}$ sendo que Rios, Barbosa \& Belasco $2010^{(23)}$ apontam também problemas crônicos no pé $(12,6 \%)$ e depressão ( $7,7 \%)$, além de estresse e esgotamento mental, o que se deve às exigências e cargas de trabalho impostas, acarretando um desequilíbrio músculo esquelético e desgaste emocional relatados por esses profissionais. ${ }^{(18,20,21,25)}$

Os profissionais de saúde referem satisfação na sua $\mathrm{QV},{ }^{(7,21,22)}$ contrastando com os relatos de presença de doenças e sintomas supracitados, mostrando a necessidade de um aprofundamento no assunto. No estudo de Silva \& Camargo 2012,(22) embora $69,7 \%$ dos profissionais tenham referido estarem satisfeitos com a QV, 30,3\% apresentaram problemas significativos que requer cuidados, justificando a implantação de programas de formulação de novas políticas públicas de saúde, por meio de planejamento e fortalecimento de ações para essa população.

O presente estudo apresenta-se de suma importância para a comunidade acadêmica por discutir e sintetizar um tema bastante relevante e questionado por todos que é a QV. Podendo servir de parâmetros para subsidiar novos trabalhos, uma vez que são escassas as publicações referentes à QV de profissionais de saúde em ambiente hospitalar, principalmente confrontando com as atividades desenvolvidas em cada unidade da instituição.

\section{CONCLUSÃO}

Evidencia-se a relevância clínica da referida pesquisa, pois dos artigos encontrados na literatura que tratam da mensuração da QV dos profissionais de saúde atuantes em ambientes hospitalares, se observou um déficit deste instrumento, sendo relacionado à situações cotidianas de exposição a diversos riscos e desencadeantes de sintomatologia psicofísicas, comprometendo a assistência como um todo. 
Se faz necessário uma reflexão em torno de algumas considerações, com ampliação das discussões e criação de programas educacionais de prevenção e controle no que se refere à saúde do trabalhador, considerando ser este a mola propulsora à conduzir e manter o pleno funcionamento das unidades hospitalares de saúde.

\section{REFERÊNCIAS}

1. Wood-Dauphinee S. Assessing quality of life in clinical research: from where have come and where are we going? J Clin Epidemiol. 1999;5(4):355-63.

2. Alleyne GAO. Health and the quality of life. Rev Panam Salud Publica. 2001; 9(1):1-6.

3. Branco JC, Giusti PH, Almeida AR, Nichorn LF. Qualidade de vida dos colaboradores de hospital universitário do Sul do Brasil. J Health Sci Inst. 2010; 28(2): 199-203.

4. Carvalho AB, Souza JC, Qualidade de vida dos profissionais de saúde do Hospital do Câncer de Campo Grande, MS. Psicólogo Informação. 2011; 15 (15):143-154.

5. Dantas RAS, Sawada NO, Malerbo MB. Pesquisas sobre qualidade de vida: revisão da produção científica das universidades públicas do Estado de São Paulo. Rev latinoam enferm. 2003;(11)4:532-38.

6. Fleck MPA, Louzada S, Xavier M, Chachamovich E, Vieira G, Santos L et al. The world health organization instrument to quality of the life (WHOQOL-1OO): Characteristics and perspectives. Ciênc. saúde coletiva 2000; 33 38.

7. Ferreira N, Oliveira J. O nível de qualidade de vida dos fisioterapeutas do complexo de saúde do Campus da Unicamp [dissertação]. Campinas, SP: Universidade Estadual de Campinas, 2008.

8. Kluthcovsky AC, Kluthcovsky F. O WHOQOLbref, um instrumento para avaliar qualidade de vida: uma revisão sistemática. Rev. psiquiatr. Rio Gd. Sul. 2009; 31(3).

9. Marcitelli CRA. Qualidade de vida no trabalho dos profissionais de saúde. Ensaios e Ciências
Biológicas, Agrárias e da Saúde. 2011; 15(4): 215-228.

1O. Sales JC, Borges CM, Alves OVM, Paes LW, Campos ACV. Qualidade de vida de três categorias profissionais da saúde em um hospital de Minas Gerais, Brasil. Rev. enferm. UFPE on line. 2010; 4(3): 1365-370.

11. Souza MA, Stancato K. Avaliação da qualidade de vida dos profissionais de saúde do Hospital das Clínicas da Unicamp. Rev enferm UFPE on line. 2011; 5(4):886-95.

12. Spiller APM, Dyniewicz AM, Glauce M, SLOMP FS. Qualidade de vida de profissionais da saúde em hospital universitário. Cogitare enferm. 2008; 13 (1):88-95.

13. Benatti MC, Nishide VM. Development and implementation of an environmental risk map for the prevention of occupational accidents in an intensive care unit at a university hospital. Rev. latinoam. enferm. 2000; 8:13-20.

14. Damas KCA, Munari DB, Siqueira KM. Cuidando do cuidador: reflexões sobre o aprendizado dessa habilidade. Rev. eletrônica enferm. 2004; 6(2): 272-278.

15. Candeias NMF, Abujamara AMD, Sabbag SN. Stress em atendentes de enfermagem. Rev. bras. saúde ocup. 2002; 2O(75):38-44.

16. Pontes FC. Trabalho participativo. 2. Ed. São Paulo: Interamericana. 2002.

17. Brasil. Ministério da Saúde. Conselho Nacional de Saúde. Princípios para NOB/RH-SUS. Brasília-DF, 2000.

18. Ansoleaga E, Toro JP, Stecher A, Godoy L, Blanch JM. Malestar psicofisiológico en profesionales de la salud pública de la región metropolitana. Psycho physiological distress among health care professionals working in Chilean public hospitals. Rev. méd. Chile. 2011; 139: 1185-1191.

19. Tong SC, Tin AS, Tan DMH, Lim JFY. The healthrelated quality of life of junior doctors. Ann Acad Med Cingapura. 2012; 444-50.

20. Oliveira LCB, Chaves-Maia EM. Saúde Psíquica dos Profissionais de Saúde em Hospitais Públicos. Rev. saúde pública. 2008; 405-413. 
21. Bastos MA, Jesus DF, Tonding JF, Gustavo AS, Urbanetto JS, Santos BRL. Qualidade de vida e cargas de trabalho do profissional enfermeiro. X Salão De Iniciação Científica-PUCRS; 2009; p. 549.

22. Silva NMM, Camargo SMPLO. Estudo comparativo da qualidade de vida dos profissionais de enfermagem que atuam no hospital público e privado no município de Bebedouro. Rev EPeQ/Fafibe on-line. 2O12; 4: 70-74.

23. Rios KA, Barbosa DA, Belasco AGS. Avaliação da qualidade de vida e depressão dos técnicos e auxiliares de enfermagem. Rev. latinoam. enferm. 2010;18(3):122-30,

24. Motke MB, Franco GP. Qualidade de vida em saúde da equipe de enfermagem da unidade de emergência de um hospital de grande porte do interior do Rio Grande do Sul. Revista Contexto \& Saúde jul./dez. 2003; p. 129-148.

25. Martínez OF, García BGDR, Cabrera CH, López CL, Tapia AM, Suárez SM. Percepción de la calidad de vida profesional de los médicos residentes de dos hospitales de distinto nivel assistencial. Medicina de Família. 2007; 2:11-18.

26. Fogaça MC, Carvalho WB, Nogueira-Martins LA. Estudo preliminar sobre a qualidade de vida de médicos e enfermeiros intensivistas pediátricos e neonatais. Rev. Esc. Enferm. USP. 2010; 44(3):708-12.

27. Kimura M, Dirley MC. Desenvolvimento e validação de uma versão reduzida do instrumento para avaliação da qualidade de vida no trabalho de enfermeiros em hospitais. 2009. Rev. Esc. Enferm. USP; 43(Esp):1044-54.

Tabela 1. Exposição dos estudos em relação à população e variáveis analisadas

(continua)

\begin{tabular}{|c|c|c|}
\hline Estudo & AMOSTRA & VARIÁVEIS ANALISADAS \\
\hline $\begin{array}{c}\text { Silva \& Camargo }{ }^{22} \\
2012\end{array}$ & $\begin{array}{c}\mathrm{N}=212 \\
4,7 \% \text { auxiliares; } 73,8 \% \\
\text { técnicos de enfermagem; } 0,5 \% \\
\text { obstetras; } 20,8 \% \text { enfermeiros }\end{array}$ & $\begin{array}{c}\text { Profissão predominante: equipe de } \\
\text { enfermagem } \\
\text { Gênero: }+ \text { > > } \widehat{~} \text { - } 88 \% \\
\text { Faixa etária: entre } 26 \text { a } 31 \text { anos } \\
\text { Instrumento: WHOQOL-bref, não foi } \\
\text { identificado os escores dos domínios. } \\
\text { Satisfação com a QV: } 69,7 \%\end{array}$ \\
\hline $\begin{array}{c}\text { Souza \& Stancato" } \\
2010\end{array}$ & $\begin{array}{c}\mathrm{N}=116 \\
27,62 \% \text { enfermeiros } \\
5,71 \% \text { fisioterapeutas } \\
12,38 \% \text { médicos } \\
2,86 \% \text { nutricionistas } \\
51,43 \% \text { técnicos de enfermagem }\end{array}$ & $\begin{array}{c}\text { Profissão predominante: equipe de } \\
\text { enfermagem } \\
\text { Gênero: } \propto>\widehat{\jmath}-78,8 \% \\
\text { Estado civil: } 44,8 \% \text { casados } \\
\text { Faixa etária: média de } 35 \text { anos } \\
\text { Instrumento utilizado: WHOQOL-bref } \\
\text { ( } \downarrow \text { domínio I, III e IV) }\end{array}$ \\
\hline
\end{tabular}


Tabela 1. Exposição dos estudos em relação à população e variáveis analisadas

(continuação)

\begin{tabular}{|c|c|c|}
\hline EsTUDO & AMOSTRA & VARIÁVEIS ANALISADAS \\
\hline $\begin{array}{l}\text { Branco et al. } \\
2010\end{array}$ & $\begin{array}{c}\mathrm{N}=3 \mathrm{O} 6 \\
11,1 \% \text { enfermeiros } \\
\text { 72,5\%técnicos de enfermagem } \\
\text { ou radiologia } \\
\text { 8,8\% auxiliar de enfermagem, } \\
5,2 \% \text { médicos } \\
1,3 \% \text { fisioterapeutas } \\
1,0 \% \text { farmacêuticos }\end{array}$ & 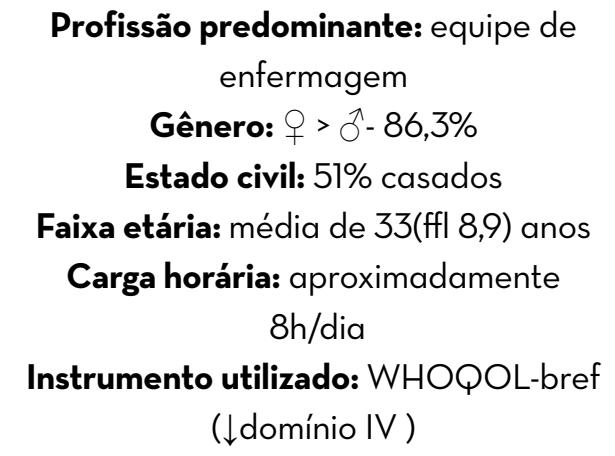 \\
\hline $\begin{array}{l}\text { Rios, Barbosa \& Belasco } 23 \\
2010\end{array}$ & $\begin{array}{c}\mathrm{N}=266 \text { técnicos e auxiliares de } \\
\text { enfermagem }\end{array}$ &  \\
\hline $\begin{array}{l}\text { Ferreira \& Oliveira }{ }^{7} \\
\qquad 2008\end{array}$ & $\mathrm{~N}=80$ fisioterapeutas & $\begin{array}{l}\text { Profissão predominante: fisioterapeutas } \\
\text { Gênero: }+>\hat{-} \text { - } 81,25 \% \\
\text { Estado civil: } 46,9 \% \text { casados } \\
\text { Faixa etária: } 45,31 \% \text { entre } 20 \text { e } 30 \text { anos } \\
\text { Carga horária: } 50 \% \text { atuam de } 21-40 \mathrm{~h} / \\
\text { semanais } \\
\text { Nível educacional: } 70,31 \% \text { cursando ou } \\
\text { cursaram mestrado e/ou doutorado } \\
\text { Instrumento utilizado: WHOQOL-bref } \\
\text { ( } \downarrow \text { domínio III) } \\
\text { Satisfação com a QV: boa ou muito boa } \\
\text { (82,21\%) e } 71,87 \% \text { satisfeito ou muito } \\
\text { satisfeito com sua saúde } \\
\text { Sintomas: } 22 \text { referiram dores na coluna }\end{array}$ \\
\hline
\end{tabular}


Tabela 1. Exposição dos estudos em relação à população e variáveis analisadas

(continuação)

\begin{tabular}{|c|c|c|}
\hline ESTUDO & AMOSTRA & VARIÁVEIS ANALISADAS \\
\hline $\begin{array}{c}\text { Motcke \& Franco }{ }^{24} \\
2003\end{array}$ & $\begin{array}{c}\mathrm{N}=38 \\
\mathrm{O} 4 \text { enfermeiros } \\
11 \text { técnicos de Enfermagem } \\
23 \text { auxiliares de Enfermagem }\end{array}$ & $\begin{array}{c}\text { Profissão predominante: auxiliares de } \\
\text { enfermagem } \\
\text { Gênero: }+>\widehat{\sigma}-71 \% \\
\text { Estado civil: } 57,9 \% \text { casados } \\
\text { Faixa etária: média de } 34 \text { anos } \\
\text { Unidade de atuação: emergência } \\
\text { Tempo de atuação: média de } 13 \text { anos } \\
\text { Instrumento utilizado: SF-36 * média } \\
\text { de } 50 \% \text { no aspecto físico } \\
\text { Sintomas: dor física }\end{array}$ \\
\hline $\begin{array}{c}\text { Spiller, Dyniewicz \& Slomp }{ }^{12} \\
2008\end{array}$ & $\begin{array}{c}\mathrm{N}=109 \\
89 \text { enfermeiros } \\
14 \text { fisioterapeutas } \\
\text { O6 nutricionistas }\end{array}$ & $\begin{array}{c}\text { Profissão predominante: enfermagem } \\
\text { Gênero: } q>\widehat{\jmath}-94,50 \% \\
\text { Estado civil: } 51,34 \% \text { casados } \\
\text { Faixa etária: } 20 \text { a } 35 \text { anos } \\
\text { Carga horária: } 44,03 \% \text { trabalham } \\
\text { sob jornada dupla ou tripla, em média } \\
\text { mais de } 9 \text { horas/dia. } \\
\text { Nível educacional: } 80,74 \% \text { possuem } \\
\text { especialização } \\
\text { Instrumento utilizado: WHOQOL- } \\
\text { bref( } \downarrow \text { domínio I) } \\
\text { Satisfação com a QV:tbaixa de } \\
\text { satisfação em todos os domínios }\end{array}$ \\
\hline $\begin{array}{c}\text { Oliveira \& Chaves-Maia }{ }^{20} \\
2008\end{array}$ & $\begin{array}{c}\mathrm{N}=95 \\
33,6 \% \text { enfermeiros } \\
28,4 \% \text { médicos } \\
16,8 \% \text { nutricionistas } \\
15,7 \% \text { assistentes sociais } \\
5,2 \% \text { psicólogos }\end{array}$ & 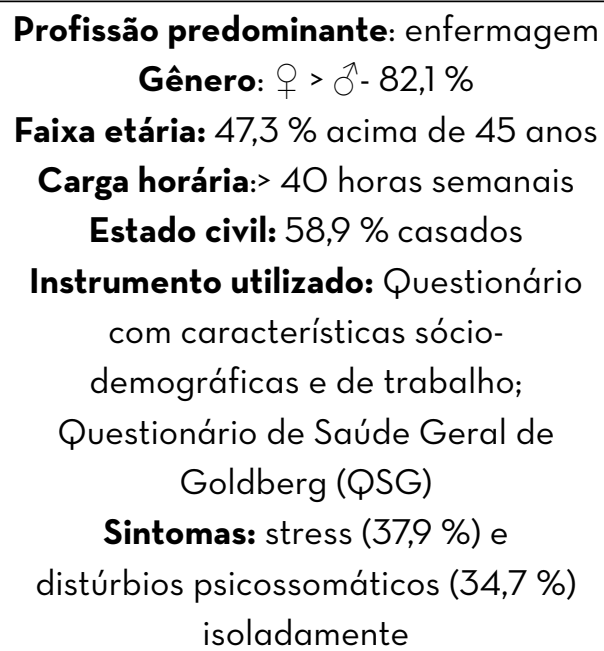 \\
\hline
\end{tabular}


Tabela 1. Exposição dos estudos em relação à população e variáveis analisadas

(continuação)

\begin{tabular}{|c|c|c|}
\hline ESTUDO & AMOSTRA & VARIÁVEIS ANALISADAS \\
\hline $\begin{array}{c}\text { Bastos et al. }{ }^{21} \\
2009\end{array}$ & $\mathrm{~N}=91$ enfermeiros &  \\
\hline $\begin{array}{c}\text { Carvalho \& Souza }{ }^{4} \\
2011\end{array}$ & $\begin{array}{l}\mathrm{N}=66 \\
\text { médicos, auxiliares e técnicos } \\
\text { de enfermagem, enfermeiros, } \\
\text { técnicos de radiodiagnóstico, } \\
\text { fisioterapeutas, farmacêuticos, } \\
\text { bioquímicos, nutricionistas, } \\
\text { assistente social, psicólogo, } \\
\text { terapeuta ocupacional e física- } \\
\text { médica }\end{array}$ &  \\
\hline $\begin{array}{c}\text { Kimura \& Dirley }{ }^{27} \\
2009\end{array}$ & $\mathrm{~N}=348$ enfermeiros & 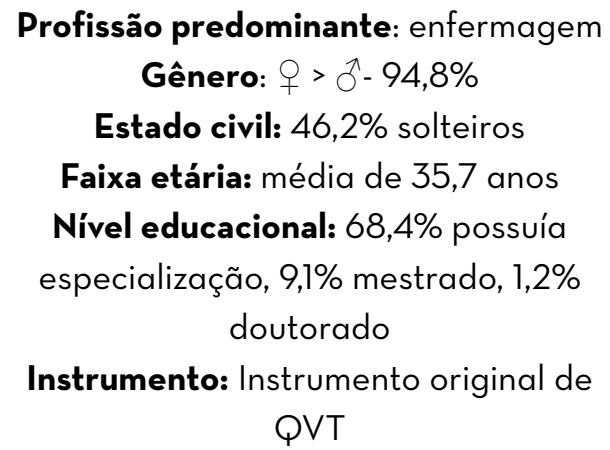 \\
\hline $\begin{array}{c}\text { Sales et al. }{ }^{10} \\
2010\end{array}$ & $\begin{array}{c}\mathrm{N}=27 \\
13 \text { enfermeiros } \\
7 \text { fisioterapeutas } \\
7 \text { médicos }\end{array}$ & $\begin{array}{l}\text { Profissão predominante: enfermagem } \\
\text { Gênero: }+>\widehat{0}-74,1 \% \\
\text { Estado civil: } 51,9 \% \text { solteiros } \\
\text { Nível educacional: } 70,1 \% \text { possui } \\
\text { especialização } \\
\text { Unidade de atuação: enfermarias } \\
\text { Instrumento utilizado: WHOQoL-bref } \\
\text { ( } \downarrow \text { domínios I e II) } \\
\text { Satisfação com QV: baixa } Q V \text { nas três } \\
\text { categorias }\end{array}$ \\
\hline
\end{tabular}


Tabela 1. Exposição dos estudos em relação à população e variáveis analisadas

(conclusão)

\begin{tabular}{|c|c|c|}
\hline ESTUDO & AMOSTRA & VARIÁVEIS ANALISADAS \\
\hline $\begin{array}{c}\text { Fogaça, Carvalho \& Nogueira- } \\
\text { Martins } \\
2010 \\
2010\end{array}$ & $\begin{array}{c}\mathrm{N}=57 \\
37 \text { médicos } \\
20 \text { enfermeiros }\end{array}$ & 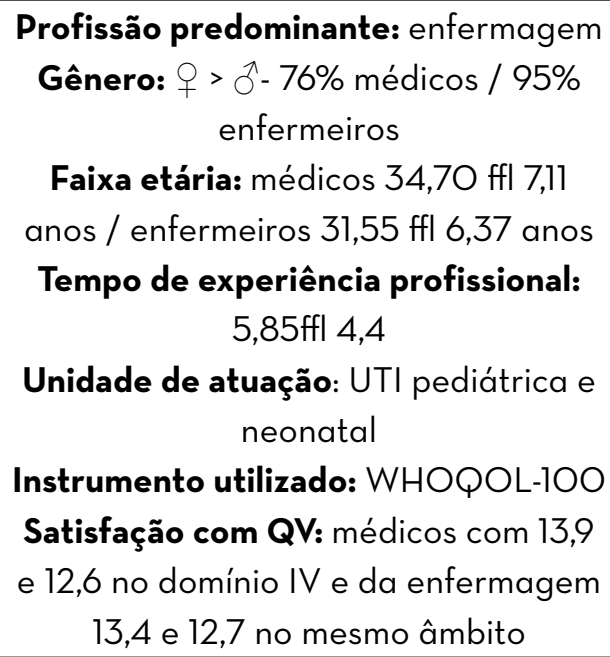 \\
\hline $\begin{array}{l}\text { Ansoelaga et al. }{ }^{18} \\
2011\end{array}$ & $\begin{array}{c}\mathrm{N}=190 \\
80 \text { médicos } \\
110 \text { enfermeiros }\end{array}$ & $\begin{array}{c}\text { Profissão predominante: equipe de } \\
\text { enfermagem } \\
\text { Gênero: } 61 \% \text { dos médicos }+<\widehat{0} ; 95 \% \\
\text { dos enfermeiros } q>\widehat{O} \\
\text { Faixa etária: idade média para } \\
\text { médicos } 46 \text { anos e } 39 \text { anos para os } \\
\text { enfermeiros } \\
\text { Instrumento utilizado: Questionário } \\
\text { de qualidade de vida laboral projeto } \\
\text { Kofarips } \\
\text { Sintomas: desgaste emocional- } \\
\text { 33\% médicos e } 44 \% \text { enfermeiros; } \\
\text { esgotamento físico -36\% dos médicos } \\
\text { e } 54 \% \text { enfermeiros }\end{array}$ \\
\hline $\begin{array}{c}\text { Tong et al. }{ }^{19} \\
2012\end{array}$ & $\begin{array}{c}\mathrm{N}=185 \\
88 \text { médicos residentes } \\
97 \text { médicos experientes }\end{array}$ & $\begin{array}{c}\text { Profissão predominante: médicos } \\
\text { Gênero: }+>\hat{~ m e ́ d i c o s ~ j o v e n s ; ~}+<\hat{0} \\
\text { médicos experientes } \\
\text { Faixa etária: média de } 33,6 \text { anos (DP } \\
8,1) \\
\text { Instrumento utilizado: SF-36v } 2 \text { - } \\
\text { médicos jovens } \\
\text { apresentaram baixos escores } \\
\text { comparados aos médicos experientes } \\
\text { em todos os domínios, exceto } \\
\text { capacidade funcional }\end{array}$ \\
\hline $\begin{array}{c}\text { Martinez et al. }{ }^{25} \\
2007\end{array}$ & $\mathrm{~N}=99$ médicos & 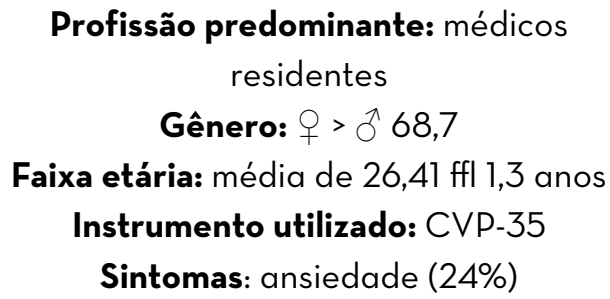 \\
\hline
\end{tabular}

